\title{
Drenaje ácido de minas y su influencia en ecosistemas asociados al yacimiento Santa Lucía, Cuba.
}

Acid mine drainage and their influence in ecosystems associated to Santa Lucia ore deposit, Cuba.

Drenagem azeda de minas e a influência nos ecossistemas associados a deposito mineral Santa Lucía, Cuba.

Damaris Gallardo Martínez / Ministerio de Ciencia Tecnología y Medio Ambiente, Cuba / regulatoriapr@citmapri.gob.cu

Noel Bruguera Amarán / Ministerio de Ciencia Tecnología y Medio Ambiente, Cuba / noel@ecovida.cu

José Antonio Díaz Duque / Universidad Tecnológica de La Habana José Antonio Echeverría, Cuba /iaduque@civil.cujae.edu.cu

lleana Cabrera Díaz / Centro de Investigaciones para la Industria Minero-Metalúgica, Cuba / ileana@cipimm.minem.cu

Recibido: 1/7/2020 Aceptado: 24/10/2020 Publicado: 30/12/2020

\section{RESUMEN}

En las actividades mineras realizadas en yacimientos sulfurosos ocurren procesos que inciden en los ecosistemas. El presente trabajo tuvo como objetivo evaluar el comportamiento de los impactos negativos provocados por el drenaje ácido de minas en el medio ambiente, tomándose como caso de estudio al yacimiento Santa Lucía del municipio Minas de Matahambre, Pinar del Río, Cuba. Las muestras fueron tomadas en la cantera abierta del yacimiento, la salida del antiguo socavón y los escurrimientos superficiales provenientes de la mina, que drenan al río Santa Lucía. Se realizó una valoración de las causas y los mecanismos de formación del drenaje ácido de minas. Se determinó su incidencia negativa en el deterioro de los ecosistemas asociados a la explotación de esos yacimientos, y se le identificó como la causa fundamental de contaminación por metales pesados en el yacimiento objeto de estudio, debido a la oxidación biótica y abiótica de los sulfuros. A partir de los impactos identificados, se diseñó el mecanismo de formación del drenaje ácido de minas en los ecosistemas. Las alternativas desarrolladas para la neutralización y tratamiento del drenaje ácido de minas, en el yacimiento Santa Lucía, son una propuesta de gestión responsable para los tomadores de decisiones.

Palabras clave: impactos ambientales, minería, yacimientos sulfurosos 


\begin{abstract}
In mining activities developed in sulfide deposits there happen processes that influence in the ecosystems, the objective of the present work is to evaluate the negative impacts provoked by the acid mine drainage in the environment, using as case of study the location of Santa Lucía, Pinar del Rio province, Cuba. The samples were taken in the open quarry of the location, the exit of the old tunnel and the superficial glides coming from the mine that drain to Santa Lucia river. It was carried out a valuation of causes and the mechanisms of formation of the mine acid drainage. Its negative incidence was determined in the deterioration of the ecosystems associated to the exploitation of these deposits. It was identified as the main cause of heavy metals contamination on the studied deposit by the biotic and abiotic oxidation of the sulfides. Starting from the identified impacts, the mechanism formation of the acid mine drainage was designed in the ecosystems. The alternatives developed for the neutralization and treatment of the mine acid drainage in Santa Lucía deposit, are a proposal of responsible management for decisors.
\end{abstract}

Keywords: environmental impacts, mining, sulfide deposits

\title{
RESUMO
}

Nas atividades mineiras levadas a cabo em locais sulfurosos que eles acontecem processos que imprensam nos ecossistemas, para o que o trabalho presente tem como objetivo para avaliar o comportamento no ambiente dos impactos negativos provocado pela drenagem azeda de minas, enquanto usando como caso de estudo o local Santa Lúcia, municipalidade Minas de Matahambre, Pinar del Rio, Cuba. As amostras foram levadas na pedreira aberto do local, a saída do túnel velho e os deslizamentos superficiais que vêm da mina que drenam para o rio San Lúcia. Ele foi levado fora uma estimação das causas e os mecanismos de formação da drenagem azeda de minas. A incidência negativa deles era determinada na deterioração dos ecossistemas associado à exploração destes locais e foi identificado como a causa fundamental de contaminação por metais pesados no objeto de estudo de local pelo biótico de oxidação e abiótico dos sulfúreos. A partir dos impactos identificados, a formação de mecanismo da drenagem azeda de minas foi projetada nos ecossistemas. Foram desenvolvidas alternativas para a neutralização e tratamento da drenagem azeda de minas no local Santa Lúcia, como uma proposta de administração responsável para os sacado de decisões.

Palavras chave: depósitos dos sulfúreos, impactos ambientais, minando

\section{INTRODUCCIÓN}

El drenaje ácido, producto de la actividad minera metálica, es una de las principales fuentes de contaminación de las aguas superficiales y subterráneas en el mundo. Este problema puede persistir durante décadas e incluso cientos de años, una vez finalizado el ciclo productivo, por lo que se debe prevenir su formación y aplicar el tratamiento más adecuado cuando se ha formado (Cabrera, 2015).

El drenaje ácido de mina, generado en actividades mineras de yacimientos de sulfuros metálicos, causa grandes problemas 
ambientales por su alto potencial de contaminación para los recursos hídricos superficiales y subterráneos. Se caracteriza por sus bajos valores de $\mathrm{pH}$ y un alto contenido en $\mathrm{mg} / \mathrm{l}$ de sulfatos y metales hierro (Fe), aluminio (Al), manganeso ( $\mathrm{Mn})$, zinc $(\mathrm{Zn})$, cobre $(\mathrm{Cu})$, plomo $(\mathrm{Pb})$, mercurio $(\mathrm{Hg})$, cadmio $(\mathrm{Cd})$, níquel ( $\mathrm{Ni})-$, nocivos para los ecosistemas (Gallardo et al., 2016).

Más del $60 \%$ de los materiales en el mundo se extraen mediante la modalidad de minería de superficie. El laboreo minero provoca la devastación de los ecosistemas donde se originan impactos ambientales negativos como la deforestación, la pérdida de la biodiversidad, la contaminación atmosférica, de las aguas terrestres, marinas y de los suelos, por elementos geodisponibles (Gallardo et al., 2013).

El sector minero se considera el mayor contaminante de las aguas terrestres. En los ecosistemas acuíferos se encuentran cargas adicionales de elementos potencialmente tóxicos, tales como: $\mathrm{Cu}, \mathrm{Zn}, \mathrm{Pb}, \mathrm{Cd}$, plata $(\mathrm{Ag})$, arsénico (As) y $\mathrm{Mn}$, que evidencian la contaminación.

En las regiones mineras el origen de los yacimientos, la tecnología de procesamiento y el desarrollo de las políticas ambientales que se aplican, son determinantes para la mitigación de los problemas ambientales provocados en los diferentes ecosistemas.

En la actualidad, el desarrollo minero está sujeto a la evaluación de indicadores que facilitan el trabajo, la gestión minera, el crecimiento económico, las compensaciones y el desarrollo. Estos son necesarios para evaluar la sostenibilidad de dichos procesos y permitir a los tomadores de decisiones encausar la gestión a desarrollar (Alfonso et al., 2019).
El yacimiento Santa Lucía, constituido por dos tipos de menas, las pirito-polimetálicas y las barito-polimetálicas, ha sido durante años objeto de una explotación minera intensiva que ha ocasionado impactos adversos al medio. En sus inicios se desarrolló la explotación para la obtención de ácido sulfúrico a partir de la pirita y, posteriormente, para la obtención de oro y plata. Las prácticas de mitigación no fueron efectivas; esto provocó problemas ambientales que se han agudizado con el tiempo. Dentro de él se encuentra la cantera abierta para la explotación del yacimiento, sin recuperar, por lo que permanecen afloramientos minerales de características sulfurosas. Estos impactos negativos bajo la acción de los agentes meteorizantes provocan la removilización de numerosos elementos contaminantes que pasan a las aguas y al suelo (Gallardo, 2018).

Una de las principales causas que provoca la contaminación en las actividades mineras, asociadas a estos tipos de yacimientos, es el drenaje ácido de mina, que no es más que el incremento de la generación de ácidos con la participación además de los procesos mineros, al destaparse el depósito mineral para su explotación. Es una contaminación de carácter inorgánico multicausal que se produce a partir de la oxidación de minerales sulfurosos y lixiviación de metales asociados, provenientes de las rocas sulfurosas cuando son expuestas al agua y al aire (Cabrera, 2015).

La investigación ejecutada identificó como problema científico que los impactos ambientales provocados por la actividad minero-metalúrgica asociada a los yacimientos sulfurosos, en la región de Santa Lucía, no habían sido evaluados con un enfoque ecosistémico, para permitir minimizar sus efectos. De ahí que se formuló como objetivo general, desarrollar 
un modelo de gestión ambiental integral con un enfoque ecosistémico para la recuperación de los impactos negativos de la actividad minero-metalúrgica, asociada a los yacimientos sulfurosos en Santa Lucía; derivándose como objetivos específicos los siguientes: evaluar el comportamiento en el medio ambiente de los impactos negativos provocados por el drenaje ácido de mina, tomándose como caso de estudio al yacimiento Santa Lucía; determinar los principales procesos que intervienen en la contaminación de las aguas terrestres por metales pesados en la región de estudio; diseñar el modelo de gestión ambiental integral para la recuperación de los impactos negativos de la actividad minerometalúrgica asociada a los yacimientos sulfurosos, en Santa Lucía; y validar el modelo de gestión diseñado en el nuevo proceso inversionista que se desarrolla en la región para la explotación de plomo y zinc, a partir de estos yacimientos sulfurosos.

El presente trabajo tiene como propósito fundamental abordar el primero de estos objetivos específicos.

\section{MATERIALES Y MÉTODOS}

Para lograr el objetivo propuesto fueron utilizados diferentes métodos. La observación se empleó para obtener la información primaria del área de estudio y las referencias disponibles sobre anteriores, descritos en estos procesos y por otros autores. El diseño experimental fue amparado por un proyecto de I+D del Centro de Investigaciones para la Industria Minera Metalúrgica y la Delegación Territorial del Ministerio de Ciencia, Tecnología y Medio Ambiente, en Pinar del Río. La valoración se realizó con las muestras tomadas en tres puntos claves: la cantera abierta del yacimiento, la salida del antiguo socavón y los escurrimientos superficiales provenientes de la mina, que drenan al río Santa Lucía (Alonso et al., 2010).

Partiendo de la aplicación del método geológico de itinerarios irregulares, se describieron un total de ocho puntos, tomados directamente en el terreno; se tomaron 11 muestras de aguas residuales, sedimentos y minerales. La localización georreferenciada se realizó teniendo en cuenta, como factor predominante para la selección, la extensión de la contaminación que se observó in situ en cada zona de interés, en dos fases investigativas; y los periodos lluviosos y poco lluviosos (tabla 1).

Una vez que se seleccionaron las muestras, los sólidos se secaron con posterioridad en el laboratorio de mineralogía del Departamento de caracterización de minerales a temperatura ambiente, y luego se pulverizaron al $100 \%$ por debajo del tamiz de $0.074 \mathrm{~mm}$ (200 mallas), con el objetivo de realizar los análisis químicos y mineralógicos correspondientes. Por otra parte, se realizaron determinaciones del $\mathrm{pH}$ a las soluciones acuosas que proceden de las muestras sólidas (sedimentos) y los residuales líquidos colectados.

\section{Principales pruebas realizadas}

Los análisis químicos de elementos mayoritarios y trazas se determinaron según los métodos de disolución ácida y fusión, con metaborato de litio para la preparación de las muestras. Se empleó un Espectrómetro de Emisión Atómica con Plasma Inductivamente Acoplado (ICP-AES), modelo Spectroflame, de la firma SPECTRO de Alemania. 
Tabla 1. Descripción macroscópica de las muestras (sedimentos, sales, residuales líquidos y aguas de río) cercanas al yacimiento Santa Lucía, con sus correspondientes coordenadas GPS.

\begin{tabular}{|c|c|}
\hline CÓDIGO & Selección de muestras y localización del objeto de investigación \\
\hline M-1 & $\begin{array}{l}\text { Muestra del frente de cantera, donde afloran las rocas del mineral polimetálico primario del } \\
\text { depósito Santa Lucía (X: } 195945 ; \text { Y: } 316 \text { 110) }\end{array}$ \\
\hline$M-1 A$ & Residual líquido que procede de la escorrentía dentro de la propia mina Santa Lucía \\
\hline M-2 & $\begin{array}{l}\text { Sedimento del escurrimiento en el afloramineto mineral dentro de la propia mina Santa Lucía } \\
\text { (X: } 195945 ; \text { Y: } 316 \text { 110) }\end{array}$ \\
\hline $\mathrm{M}-2 \mathrm{~A}$ & Residual líquido colectado a la salida del socavón de la mina Santa Lucía \\
\hline M-3 & $\begin{array}{l}\text { Sedimento del escurrimiento del embalse que procede de la solución acuosa de la muestra-1A, de } \\
\text { la mina Santa Lucía (X: } 194 \text { 149; Y: } 315 \text { 153) }\end{array}$ \\
\hline$M-3 A$ & Residual líquido que proviene de la escorrentía del lado del socavón de la mina Santa Lucía \\
\hline M-4 & $\begin{array}{l}\text { Sales ferrosas oxidadas -de color verde-amarillentas- formadas a la salida del socavón de Santa } \\
\text { Lucía (X:195 960; Y: } 316 \text { 115) }\end{array}$ \\
\hline M-5 & Sedimento de la salida del socavón de la mina Santa Lucía (X: 195 960; Y: 316 115) \\
\hline M-6 & Sedimento recolectado a un lado del socavón de Santa Lucía (X: 195 965; Y: 316 113) \\
\hline M-9A & Muestra de agua del efluente del río Santa Lucía tomada in situ (X: 197 513; Y: 314 945) \\
\hline M-10A & Muestra de agua del propio río Santa Lucía, tomada in situ (X: 197 513; Y: 314 945) \\
\hline
\end{tabular}

Fuente: Elaboración propia.

La prueba de movilidad de metales en desechos sólidos, en presencia de agua meteórica, se realizó para confirmar la posibilidad de lixiviación de metales en los desechos sólidos, en concentraciones geodisponibles; para ello se utilizó el procedimiento técnico Meteoric Water Mobility Procedure (MWMP) empleado por el Centro de Investigaciones para la Industria Minera Metalúrgica.

La prueba estática estándar de conteo ácido-base permitió evaluar la capacidad de neutralización de la muestra, al detreminar el ácido consumido durante la digestión con un exceso de este. Se comparó el resultado con el potencial ácido a partir del cálculo del análisis del azufre sulfúrico. Dicha prueba se realizó por el procedimiento analítico desarrollado por Sobeck, y descrito por Price et al. (1997) y Lawrence \& Wang (1997).
La prueba química de neutralización de aguas ácidas generadoras de drenaje ácido de mina se realizó con el propósito de neutralizar las aguas ácidas, provenientes del drenaje ácido de mina, así como para comprobar la efectividad y viabilidad del agente neutralizante. Para las pruebas se tomaron $500 \mathrm{ml}$ de cada efluente en el lugar muestreado y se trataron con pulpas al $20 \%$ de carbonato de calcio (99\% pureza), hasta alcanzar un pH entre 6 y 6.5. Posteriormente, seles añadió soluciones de hidróxido de calcio (60\% pureza) y pulpas de óxido de calcio (60\%), se mantiene la agitación continua hasta alcanzar un valor de $\mathrm{pH}$ de 9 y 9.5, el cual se corresponde con el índice de acidez que se requiere para precipitar soluciones que contienen los diferentes metales. Para la comparación de los mismos se empleó la norma cubana NC27:2012 Vertimiento de 
las aguas residuales a las aguas terrestres y el alcantarillado. Especificaciones (Oficina Nacional de Normalización [ONN], 2012).

La neutralización/precipitación con cal (como $\left.\mathrm{CaO} \circ \mathrm{Ca}(\mathrm{OH})_{2}\right)$, en la práctica mundial, se reporta como el tratamiento más eficiente a nivel industrial. Esta alternativa logra la neutralización de la acidez y la precipitación de los metales. A medida que aumenta el pH, los aniones se tornan más disponibles para su precipitación.

El método de las diluciones sucesivas o seriadas (end point), desarrollado por Silverman \& Lundgren (1959), se empleó para el análisis de la oxidación biótica con el propósito de determinar la presencia de microorganismos en las muestras de la mina Santa Lucía, los cuales pueden incidir en la oxidación de la pirita. En tal sentido, se encuentran presentes los elementos nutrientes, energéticos y la acidez requerida para el crecimiento celular. Está formado por la mezcla de dos soluciones, la solución 1 , que contiene varias sales, constituye el $70 \%$ del medio y se esteriliza en autoclave; y la solución 2, compuesta por sulfato ferroso $\left(\mathrm{FeSO}_{4}\right)$, que representa el $30 \%$ restante, se esteriliza por filtración. Una vez preparadas las soluciones, se mezclan y se ajusta la acidez con ácido sulfúrico $\left(\mathrm{H}_{2} \mathrm{SO}_{4}\right)$ a $\mathrm{pH}$ 2.0. Este medio de cultivo se utilizó en la preparación de las pulpas objeto de prueba.

Para realizar el aislamiento, se prepararon tres juegos de nueve tubos de ensayos; los que se esterilizan y se identifican con números consecutivos. En cada tubo de ensayo se vierten $9 \mathrm{ml}$ de medio $9 \mathrm{k}$. Se toma $1 \mathrm{ml}$ de agua de la mina y se mezcla con los $9 \mathrm{ml}$ de medio del primer tubo de ensayos, se agita burbujeando ligeramente, y se extrae $1 \mathrm{ml}$ de esta primera dilución, que se deposita en el segundo tubo de ensayos; se repite la operación de mezcla y burbujeo anterior, y se extrae $1 \mathrm{ml}$ de la segunda dilución, el que se deposita en el tercer tubo de ensayo; y así sucesivamente hasta llegar al último tubo.

Con cada juego de tubos se repitieron las mismas operaciones. A cada uno de los tubos de ensayos de los tres juegos que contenían las diluciones, se les situó un tapón de gasa y algodón y se colocaron en la zaranda orbital termostática, con una frecuencia de rotación de $1 \mathrm{~s}^{-1}$ (60 rpm) y temperatura de $30^{\circ} \mathrm{C}$. Las muestras se mantuvieron en la zaranda durante el período de duración de la prueba; se realizaron el control y la observación diarios. Para la adaptación del microorganismo al concentrado, se añadió $1 \mathrm{~g}$ de mineral a $10 \mathrm{ml}$ de medio $9 \mathrm{k}$ con $1 \mathrm{ml}$ del cultivo del microorganismo aislado.

Los ensayos análiticos ambientales estuvieron orientados a la evaluación científico-tecnológica de la valoración ecosistémica realizada, y a la identificación del mecanismo de formación del drenaje ácido de mina y su impacto en los ecosistemas.

Los métodos teóricos utilizados (analíticosintético, deducción-inducción, hipotéticodeductivo, sistémico y modelación) permitieron comprender y explicar los impactos del drenaje ácido de mina en los ecosistemas presentes en la región de estudio.

\section{RESULTADOS Y DISCUSIÓN}

Los minerales sulfurosos caracterizan el ambiente geológico de la región, la exposición de roca sulfurosa reactiva al aire y al agua, como resultado de los inadecuados procesos de cierre de minas, acelera la velocidad de generación de ácido y ocasiona impactos ambientales negativos.

Desde el punto de vista ecosistémico, el yacimiento Santa Lucía se encuentra en un 
ecosistema montañoso. La cantera y los afloramientos están fundamentalmente en las áreas de explotación y los procesos climatológicos, como las precipitaciones, facilitan los escurrimientos superficiales y la incorporación del drenaje ácido a los ecosistemas de agua dulce, en este caso al río Santa Lucía, que transporta los contaminantes a la zona costera donde desemboca, lo cual afecta al manglar.

En la tabla 2 se muestran los resultados de los análisis químicos mayoritarios de las muestras tomadas. En la muestra de roca primaria M-1 se refleja el quimismo característico de las menas pirito-polimetálicas de $\mathrm{Pb}, \mathrm{Zn}$ y $\mathrm{Ba}$, de Santa Lucía. El resto de las muestras presentan la composición química típica de los sedimentos formados durante la intensa contaminación ácida existente. Estos análisis evidencian la contaminación de la región, producto a la exposición de los yacimientos por largos periodos de tiempo.

En los análisis de las muestras tomadas en Santa Lucía (M-2, M-3, M-5, M-6, M-7) existe una tendencia a la disminución de los contenidos de $\mathrm{Pb}$, Fe y Zn. Comparados con la roca primaria (M-1), este comportamiento se justifica por la ocurrencia de intensos procesos de drenaje ácido de mina.

La evaluación de la composición elemental de la muestra M-4 (sales a la salida del socavón) arroja un incremento del contenido de FeO, de forma significativa, que alcanza un valor máximo del 20.69\%. De forma análoga,

Tabla 2. Resultados de los análisis químicos de elementos mayoritarios de las muestras tomadas.

\begin{tabular}{ccccccccccc}
\hline Muestra & $\% \mathrm{SiO}_{2}$ & $\% \mathrm{Al}_{2} \mathrm{O}_{3}$ & $\% \mathrm{FeO}$ & $\% \mathrm{MnO}$ & $\% \mathrm{PbO}_{2}$ & $\% \mathrm{ZnO}$ & $\% \mathrm{BaO}$ & $\% \mathrm{Fe}_{2} \mathrm{O}_{3}$ & $\% \mathrm{CaO}$ & $\% \mathrm{PPI}$ \\
\hline M-1 & 30.31 & 0.32 & 15.57 & 0.19 & 6.32 & 2.10 & 2.42 & 0.56 & 0.25 & 40.16 \\
M-2 & 42.46 & 0.56 & 6.59 & 0.05 & 1.56 & 0.28 & 4.07 & 8.13 & 1.21 & 32.26 \\
M-3 & 54.48 & 0.66 & 2.99 & 0.04 & 0.86 & 0.27 & 8.04 & 14.45 & 0.23 & 15.46 \\
M-4 & N.D & 0.08 & 20.69 & 0.16 & 0.06 & 5.01 & N.D & N.D & 0.01 & 74.18 \\
M-5 & 76.01 & 0.49 & 1.36 & 0.02 & 0.07 & 0.50 & 5.01 & 5.48 & 0.10 & 8.02 \\
M-6 & 67.53 & 0.64 & 1.02 & 0.02 & 0.26 & 0.31 & 6.56 & 13.21 & 0.07 & 7.62 \\
\hline
\end{tabular}

Fuente: Elaboración propia

se incrementa la pérdida por ignición (PPI) de los componentes volátiles fundamentales: $\mathrm{SO}_{3} ; \mathrm{H}_{2} \mathrm{O}^{+}$. Para el $\mathrm{ZnO}$ se obtuvo un valor anómalo de 5.01\%. Estos resultados son elevados al compararlos con los obtenidos en la muestra M-1. También presenta una coloración amarillo-verdosa; este proceso indica la presencia de bacterias catalizadoras de la oxidación.
En la tabla 3 se muestran los resultados representados en los análisis físico-químicos de elementos contaminantes, contenidos en aguas de río y drenajes líquidos (escorrentías); se comprueba el incumplimiento de la norma cubana NC27:2012 (ONN, 2012) para los elementos $\mathrm{Zn}, \mathrm{Pb}$ y $\mathrm{Fe}$; otra evidencia de la intensidad del proceso de drenaje ácido asociado al yacimiento. 
Tabla 3. Resultados de los análisis químicos de elementos contaminantes en aguas de río y drenajes líquidos (escorrentías) de las muestras tomadas.

\begin{tabular}{cccccc}
\hline \multirow{2}{*}{ Muestras } & \multicolumn{6}{c}{ Concentración de los elementos (mg/l) } \\
& Zn & Pb & Ba & Fe & S (total) \\
\hline M-1A & 6.0 & 64.9 & $<0.2$ & 14.7 & 15.3 \\
M-2A & 5.6 & 58.9 & $<0.2$ & 13.9 & 11.0 \\
M-3A & 81 & 62.3 & $<0.2$ & 14.1 & 15.7 \\
M-9A & $<1.7$ & $<0.4$ & $<0.2$ & 4.6 & 85 \\
M-10A & $<2.1$ & $<0.4$ & $<0.2$ & 4.6 & 1.4 \\
NC 27/12 & $<5.0$ & $<1.0$ & $<1.0$ & $<5.0$ & $<5.0$ \\
\hline
\end{tabular}

Fuente: Elaboración propia

El comportamiento del $\mathrm{pH}$ se explica por la presencia de iones $\mathrm{H}_{3} \mathrm{O}^{+}$y $\mathrm{S}^{2-}$, disueltos dentro de la matriz del sólido, los que al combinarse en solución acuosa y en presencia de aire, reaccionan y dan lugar a la formación de ácido sulfúrico; ello evidencia que ocurre un drenaje ácido de mina (tabla 4).

Tabla 4. Valores de pH obtenidos en las soluciones acuosas de las fases sólidas (sedimentos) de las muestras seleccionadas en Santa Lucía.

\begin{tabular}{cc}
\hline Muestra & pH \\
\hline $\mathrm{M}-1$ & 3.0 \\
$\mathrm{M}-2$ & 2.9 \\
$\mathrm{M}-3$ & 3.3 \\
$\mathrm{M}-4$ & 2.9 \\
$\mathrm{M}-5$ & 3.2 \\
$\mathrm{M}-6$ & 3.4 \\
\hline
\end{tabular}

Fuente: Elaboración propia

En las minas de yacimientos sulfurosos a las que no se les realiza un proceso de mitigación correcto por parte de los concesionarios, el drenaje ácido se convierte en una de las fuentes más importante de contaminación, principalmente de las aguas terrestres y de los suelos. La explotación minera del yacimiento Santa Lucía, con bajos índices en la mitigación de los impactos y por las características geológicas del campo mineral, originó procesos de contaminación basados en los principios antes expuestos, esto se evidencia en la figura 1. 
Figura 1. Impacto del drenaje ácido de minas en los ecosistemas próximos al yacimiento Santa Lucía, Pinar del Río, Cuba.

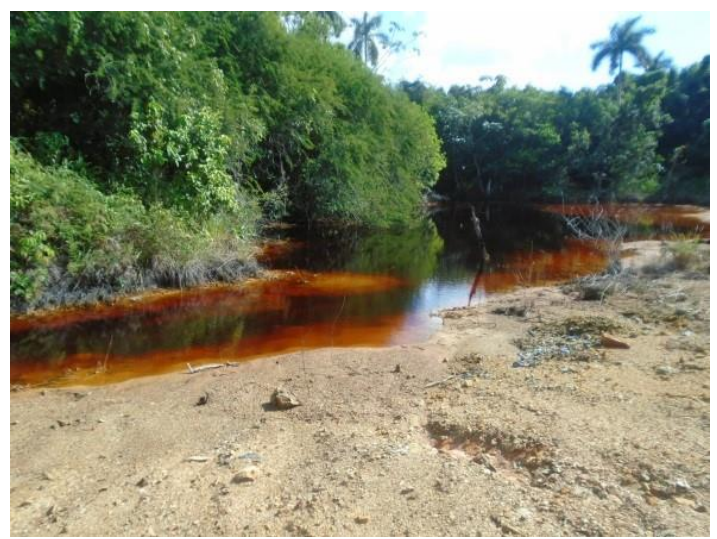

Fuente: Gallardo (2018).

Determinación de la oxidación biótica de acidófila Acidithiobacillus ferrooxidans, los sulfuros en los residuales líquidos de la anteriormente conocida como Thiobacillus mina Santa Lucía.

La acidez y los metales disueltos, detectados en las muestras de agua del drenaje de la mina Santa Lucía, constituyen indicadores de la presencia de microbiota autóctona (principalmente bacterias acidófilas), de estos yacimientos de minerales sulfurosos secundarios, tales como la pirita, el sulfuro de cobre y de zinc. A partir del análisis cuantitativo de muestras del agua de la mina se realizó el aislamiento de microorganismos acidófilos mediante el medio 9K, desarrollado por Silverman \& Lundgren (1959) y el método de las diluciones sucesivas o seriadas (end point). La presencia de microorganismos acidófilos en el agua de la mina Santa Lucía mostró, luego de los ocho días de crecimiento de la cepa, el cambio de coloración del licor, de verde a pardo rojizo, evidenciándose la presencia de hierro oxidado. Se aisló la bacteria autótrofa, aerobia, quimiolitotrófica, mesófila y ferrooxidans, identificada como AFSL 1-09, con una concentración celular en estas aguas de $105 \mathrm{cel} / \mathrm{ml}$. El crecimiento de los microorganismos se siguió por el análisis del contenido de ion $\mathrm{Fe}_{3+}$ presente en el medio de cultivo, reportado por Karavaiko et al. (1972).

Durante el proceso de oxidación del hierro se produce el crecimiento bacteriano, se incrementa la concentración celular y, con ello, el contenido de hierro (III) en los licores, corroborándose la influencia de los microorganismos en la generación del drenaje ácido de mina, en los yacimientos sulfurosos. Los resultados de las reacciones de oxidación de los sulfuros en esta zona se expresan a continuación.

\section{Oxidación abiótica de la pirita en el área de estudio.}

En contacto con la atmósfera y en presencia de agua, se produce la oxidación directa de la pirita: 
A) Oxidación de la pirita y esfalerita a sulfatos de hierro II e hidróxido de hierro III:

$$
\begin{aligned}
& \mathrm{FeS}_{2(\mathrm{~s})}+7 / 2 \mathrm{O}_{2}+2 \mathrm{H}_{2} \mathrm{O} \rightarrow \mathrm{FeSO}_{4} . \mathrm{H}_{2} \mathrm{O}_{(\mathrm{s})}+\mathrm{H}_{2} \mathrm{SO}_{4} \quad(\mathrm{pH}=2.0-3.0)(1) \\
& \text { PIRITA (MP) (ZSOMOLNOKITA GLOBULAR) } \\
& 2 \mathrm{FeSO}_{4} . \mathrm{H}_{2} \mathrm{O}_{(\mathrm{s})}+\mathrm{ZnS}_{(\mathrm{s})}+9 \mathrm{H}_{2} \mathrm{O}+3 / 2 \mathrm{O}_{2} \rightarrow \mathrm{FeSO}_{4} .4 \mathrm{H}_{2} \mathrm{O}+(\mathrm{Fe}, \mathrm{Zn}) \mathrm{SO}_{4} .6 \mathrm{H}_{2} \mathrm{O}+\mathrm{H}_{2} \mathrm{SO}_{4}(2) \\
& \text { (ZSOMOLNOKITA) (ESFALERITA) (ROZENITA FIBROSA) (BIANCHITA FIBROSA) } \\
& 2 \mathrm{FeS}_{2(\mathrm{~s})}+7 \mathrm{H}_{2} \mathrm{O}+13 / 2 \mathrm{O}_{2(\mathrm{~g})} \rightarrow 2 \mathrm{Fe}(\mathrm{OH})_{3(\mathrm{~s})}+4 \mathrm{H}_{2} \mathrm{SO}_{4} \mathrm{H}_{2} \mathrm{O}+\mathrm{O}_{2},(\mathrm{pH}=4) \text { (3) }
\end{aligned}
$$

\begin{abstract}
Es decir, se produce acidez y se liberan de un substrato inorgánico). Estos sulfatos y $\mathrm{Fe}$, junto con otros elementos que microorganismos como Acidithiobacillus en mayor o menor proporción forman parte ferrooxidans, Leptospirillum ferrooxidans de la pirita. $\quad$ y Acidithiobacillus thiooxidans, tienen

\section{Oxidación biótica de la pirita.}

La oxidación biótica de la pirita ocurre con la intervención de determinadas bacterias

su desarrollo óptimo en condiciones de $\mathrm{pH}$ ácido, que cataliza las reacciones de oxidación, y obtienen su energía de este proceso (Nordstrom \& Southam, 1997).
\end{abstract} quimiolitótrofas (aquellas que se alimentan

B) Oxidación del sulfato de hierro II - III catalizada por Thiobacillus Ferrooxidans:

\author{
$7 \mathrm{FeSO}_{4(\mathrm{~s})}+25 \mathrm{H}_{2} \mathrm{O}+\mathrm{H}_{2} \mathrm{SO}_{4}+5 \mathrm{O}_{2}(\mathrm{~g})+\mathrm{MgSO}_{4} \cdot 6 \mathrm{H}_{2} \mathrm{O}(\mathrm{s}) \rightarrow$ \\ HEXAHIDRITA \\ $\mathrm{Fe}_{2}\left(\mathrm{SO}_{4}\right)_{3} .9 \mathrm{H}_{2} \mathrm{O}_{(\mathrm{s})}+\left[\left(\mathrm{Fe}^{2+}, \mathrm{Mg}\right) \mathrm{Fe}_{4}\left[\mathrm{OH}\left(\mathrm{SO}_{4}\right)_{3}\right]_{2} .20 \mathrm{H}_{2} \mathrm{O}\right]_{(\mathrm{s})}(4)$ \\ (COQUIMBITA) (Mg - COPIAPITA)
}

Resulta relevante el aumento de la tasa de oxidación de $\mathrm{Fe}^{2+}$ a $\mathrm{Fe}^{3+}$, que constituye el factor limitante de todo el proceso de forma abiótica. Bacterias como Acidithiobacillus ferrooxidans pueden acelerar esta reacción hasta 100000 veces. Al aumentar la concentración de $\mathrm{Fe}^{3+}$ se produce un incremento de la oxidación indirecta de la pirita, que genera más $\mathrm{Fe}^{2+}$ y de nuevo se oxida a $\mathrm{Fe}^{3+}$, de manera que estas reacciones se retroalimentan en un ciclo sin fin.

\section{Resultados de las pruebas de tratamiento químico para la neutralización de las aguas ácidas producidas por la oxidación de los sulfuros en la región.}

En la tabla 5 se presentan las propiedades físico-químicas y los costos de los diferentes agentes neutralizantes empleados en las pruebas, para ofrecer criterios de selección en la propuesta tecnológica a implementar por los responsables de efectuar las medidas de remediación. 
Tabla 5. Propiedades físico-químicas y costos de los reactivos empleados en los procesos de neutralización.

\begin{tabular}{cccc}
\hline Reactivos & pH saturación & $\begin{array}{c}\text { Solubilidad del reactivo en } \\
\text { agua a } \mathbf{1 8}{ }^{\circ} \mathrm{C}(\mathbf{m g} / \mathbf{l})\end{array}$ & $\begin{array}{c}\text { Costo } \$ / \text { t de ácido } \\
\text { neutralizado }\end{array}$ \\
\hline $\mathrm{CaCO}_{3}$ & $6.0-6.5$ & 14 & $15-45$ \\
$\mathrm{CaO}$ & 12.4 & $1300-1850$ & $130-300$ \\
$\mathrm{Ca}(\mathrm{OH})_{2}$ & 12.4 & $1300-1850$ & $150-300$ \\
\hline
\end{tabular}

Fuente: Elaboración propia

El comportamiento de las pruebas de a temperatura ambiente de los minerales neutralización química empleando los portadores de elementos potencialmente diferentes agentes neutralizantes en las tóxicos sería el siguiente: FeS2 (pirita), ZnS campañas de muestreo realizadas, se (esfalerita) y PbS (galena).

reporta en la tabla 6 . El orden de lixiviación

Tabla 6. Pruebas de neutralización química realizadas para las muestras tomadas en los lugares más impactados por el drenaje ácido.

\begin{tabular}{ccccccccc}
\hline Muestra & $\begin{array}{c}\text { Agente } \\
\text { Neutral (\%) }\end{array}$ & pH & Fe & Pb & Zn & Mg & As & St \\
\hline \multirow{2}{*}{ MSTL-1 } & Cabeza & 2.5 & 13260 & 2.3 & 8030 & 990 & 13 & 12190 \\
Cantera & $\mathrm{CaCO}_{3}$ & 6.5 & 3000 & 2.0 & 1330 & 170 & 0.4 & 3080 \\
Santa Lucía & $\mathrm{Ca}(\mathrm{OH})_{2}$ & 9.5 & 0.4 & 2.0 & 0.96 & 130 & 0.4 & 550 \\
& $\mathrm{CaO}$ & 9.5 & 0.4 & 2.0 & 0.20 & 160 & 0.4 & 860 \\
& $\mathrm{Cabeza}$ & 2.9 & 14300 & 2.3 & 8480 & 570 & 16.2 & 11550 \\
MSTL-3 & $\mathrm{CaCO} 3$ & 6.5 & 3060 & 0.4 & 1380 & 170 & 0.4 & 3040 \\
Efluentes del & $\mathrm{Ca}(\mathrm{OH})_{2}$ & 9.5 & 0.78 & 0.4 & 0.45 & 0.2 & 0.4 & 420 \\
socavón & $\mathrm{CaO}$ & 9.5 & 0.46 & 0.4 & 0.20 & 0.4 & 0.4 & 620 \\
& $\mathrm{Cabeza}$ & 2.1 & 1070 & 2.3 & 1480 & 570 & 0.4 & 11980 \\
& $\mathrm{CaCO}$ & 6.5 & 1.20 & 0.4 & 460 & 170 & 0.2 & 650 \\
MSTL-4 & $\mathrm{Ca}(\mathrm{OH})_{2}$ & 9.5 & 0.60 & 0.4 & 1.50 & 0.2 & 0.2 & 570 \\
Santa Lucía & $\mathrm{CaO}$ & 9.5 & 0.46 & 0.4 & 0.20 & 0.1 & 0.2 & 700 \\
\hline
\end{tabular}

Nota: Concentraciones de elementos potencialmente tóxicos expresadas en mg/l.

Fuente: Elaboración propia 
La pirita se encuentra generalmente como fase mayoritaria en las menas pirito polimetálicas de los yacimientos estudiados; por ello que se reportan mayores concentraciones de $\mathrm{Fe}^{2+3+}$ en los residuales líquidos, seguidos en orden por los de $\mathrm{Zn}^{2+}$ y en menor cuantía el $\mathrm{Pb}^{2+}$, cuyo comportamiento está condicionado por el potencial redox de esos metales. Esta evaluación le facilita a las empresas encargadas de la recuperación de las áreas afectadas, herramientas para realizar su planificación al disponer del agente neutralizante, la nomenclatura a emplear y los costos de estos para la aplicación en la industria, y así cumplir con los límites de descarga exigidos por la norma cubana NC 27:2012 (ONN, 2012).

Los valores de $\mathrm{pH}$ ácido en las muestras seleccionadas se condicionan por la presencia de iones $\mathrm{H}_{3} \mathrm{O}_{+}$y $\mathrm{S}_{2}$ disueltos dentro de la matriz del sólido, los que al combinarse en solución acuosa y en presencia de aire, reaccionan y dan lugar a la formación de ácido sulfúrico, dada la ocurrencia de los procesos de drenaje ácido.

Las transformaciones físico-químicas que ocurren en los diferentes procesos de los yacimientos sulfurosos, destacan la importancia de la recuperación rápida de las áreas afectadas en estos procesos mineros. La exposición de esos depósitos a largos períodos de tiempo provoca la oxidación biótica y abiótica de los sulfuros, en especial la pirita, lo que agudiza la contaminación en la región, convirtiéndola en el problema ambiental más impactante para los ecosistemas asociados al yacimiento Santa Lucía (figura 2).

Figura 2. Mecanismo de formación del drenaje ácido de minas y su impacto en los ecosistemas.

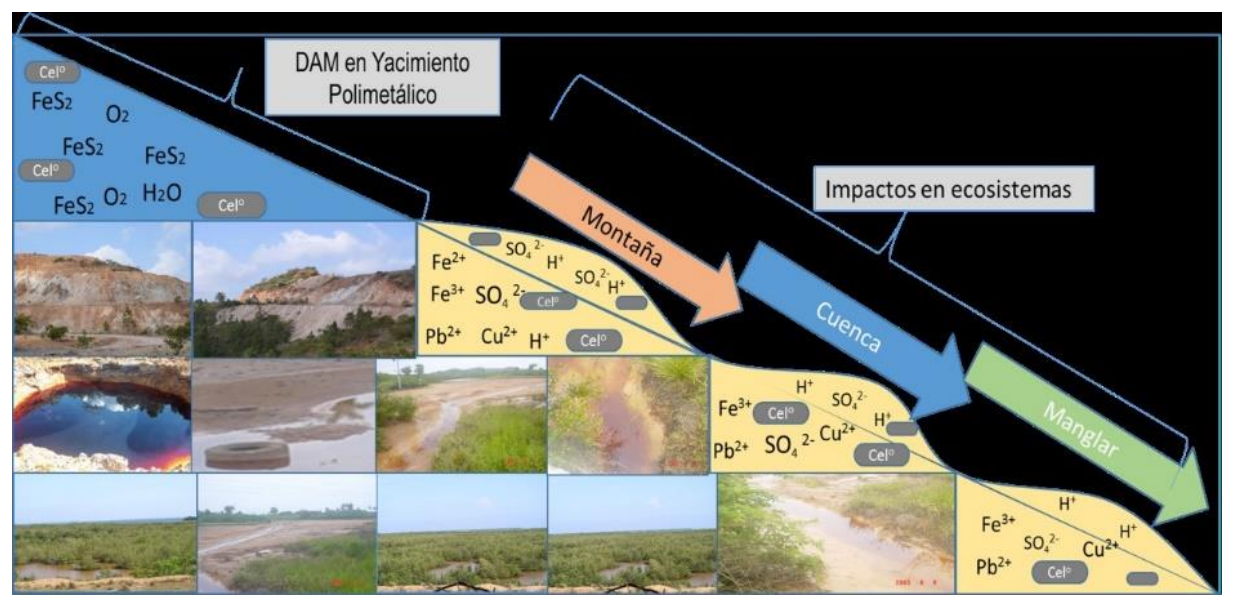

Fuente: Gallardo (2018). 
Principales acciones encaminadas a la recuperación de las áreas afectadas por el drenaje ácido de minas para el yacimiento Santa Lucía.

1) Realizar la canalización de todos los escurrimientos de la cantera, las escombreras y sitios donde los procesos como el intemperismo faciliten la ocurrencia del drenaje ácido de mina, hacia las balsas de contacto que estarán impermeabilizadas para mitigar impactos al nivel freático.

2) Valorar la cantidad necesaria de neutralizante para la remediación, de acuerdo al volumen de la balsa; lograr un $\mathrm{pH}$ entre 7 y 8.

3) Diseñar la balsa de contacto con las dimensiones que faciliten lograr el contacto necesario entre el agua residual contaminada y el agente neutralizante, en función de permitir la precipitación de los metales pesados.

4) Verter las aguas al medio ambiente solo cuando cumplan con la norma cubana NC27:2012 (ONN, 2012).

5) Evaluar los sitios afectados y utilizar técnicas de fitorremediación con especies hiperacumuladoras de metales pesados, que faciliten la descontaminación y recuperación de estos como las Brassicaceae (Cruciferae), descritas por Baker et al. (2000) y Contreras et al.
(2015)

6) Realizar los procesos de vigilancia y control ambiental en los ecosistemas para comprobar la efectividad de las medidas adoptadas.

Estas medidas son necesarias para mitigar los impactos negativos ocasionados, pues los consecionarios no realizaron la recuperación del yacimiento y en la actualidad es un pasivo ambiental; el interperismo y la exposición de la cantera abierta por tantos años ha provocado la oxidación de los sulfuros y la contaminación de la región por metales pesados.

\section{CONCLUSIONES Y RECOMENDACIONES}

Se estableció que la causa fundamental de la contaminación por metales pesados asociadas al yacimiento Santa Lucía es originada por el drenaje ácido de mina, lo que ocasiona efectos negativos en los ecosistemas expuestos como resultado de la oxidación biótica y abiótica de los sulfuros. Se diseñó el mecanismo de formación del drenaje ácido de mina y su influencia en los ecosistemas, a partir de los impactos identificados en el yacimiento Santa Lucía.

Las alternativas desarrolladas para la neutralización y tratamiento del drenaje ácido de mina, en el yacimiento Santa Lucía, son una propuesta de gestión responsable para los tomadores de decisiones.

\section{REFERENCIAS BIBLIOGRÁFICAS}

Alfonso, A., Montero, J.M., y Watson, R. (2019). Indicadores de sostenibilidad para la industria minera extractiva en Uige, Angola. Revista Minería \& Geología, 35(2), 233-251. https:// bit.ly/3eJT71R 
Alonso, J.A., Pinto, A., Cabrera, I., Cozzi, G., Gallardo, D., Valdivia, G., y Díaz, A. (2010). Principales asociaciones mineralógicas de elementos contaminantes presentes en residuales de la industria minero - metalúrgica, segunda etapa (Informe de proyecto I+D no publicado). Ministerio de Energia y Minas-Ministerio de Ciencia Técnologia y Medio Ambiente, Cuba.

Baker, A.J., Mcgrath, S.P., Reeves, R.D., \& Smith, J.A.C. (2000). Metal hyperacumulator plants: a review of the ecology and physiology of a biological resource for phytoremediation of metal-polluted soils. In N. Terry y G. Bañuelos (Eds.), Phytoremediation of Contaminated Soil and Water (pp. 85-107). Lewis Publisher.

Cabrera, I. (2015). Influencia del pasivo minero sulfometales sobre el drenaje ácido y elementos potencialmente tóxicos en el manglar [Tesis de maestría, Universidad de Pinar del Río Hermanos Saiz Montes de Oca]. Repositorio Institucional UPR. .

Contreras, L.A., Valencia, C.M., de la Fuente, N.M., Linaje, M.S., y Trejo, R. (2015). Estudio de absorción, acumulación y potencial para la remediación de suelo contaminado por plomo usando Ambrosia Ambrosioides. Revista de Investigación y Desarrollo en Ciencia y Tecnología de Alimentos, 1(1), 244-250. https://bit.ly/34DkEy7

Gallardo, D. (2018). Modelo de gestión ambiental para la actividad minero metalúrgica en los yacimientos sulfurosos del campo mineral Santa Lucía - Castellanos [Tesis doctoral no publicada]. Universidad de Pinar del Río Hermanos Saiz Montes de Oca.

Gallardo, D., Bruguera, N.C., Díaz Duque, J.A., y Cabrera, I. (2016). Impacto provocado por la minería en la zona de Santa Lucía, evaluación físico-química. Revista Minería \& Geología, 31(4), 100-120. https://bit.ly/2ImEKnS

Gallardo, D., Cabrera, I., Bruguera, N.C., Alonso, J.A., Pinto, A., \& Milián, E. (2013). Chemical physical evaluation of the superficial waters in areas with miner-metallurgic activity in Santa Lucia, Pinar del Rio. In: Farfán, H., Corvea, J.L., de Bustamente, I. \& LaMoreaux, J.W. (Eds.), Management of water resources in protected areas (pp. 293-300). Springer. https://bit.ly/38rOg4f

Karavaiko, G.I., Kuznetsov, S.I., \& Golomzik, A.I. (1972). Role of microorganism in leaching of metals from ores. Nauka.

Lawrence, R.W., \& Wang, Y. (1997, May 31-June 6). Determination of neutralization potential acid rock drainage [ponencia]. Fourth International Conference On Acid Rock Drainage. American Society of Surface Mining and Reclamation, Vancouver BC, Canada.

Nordstrom, D.K., \& Southam, G. (1997). Geomicrobiology of Sulfide Mineral Oxidation. In J.F. Banfield \& K.H. Nealson (Eds.), Geomicrobiology: Interactions between Microbes and Minerals. Reviews in Mineralogy, Vol. 35 (pp. 361-390). Mineralogical Society of America. 
Oficina Nacional de Normalización. (2012). Vertimiento de las aguas residuales a las aguas terrestres y el alcantarillado. Especificaciones. (Norma Cubana 27:2012)

Price, W.A., Morrin, K., \& Hutt, N. (1997, May 31-June 6). Guidelines for the prediction of acid rock drainage, part /I [ponencia]. Fourth International Conference On Acid Rock Drainage. American Society of Surface Mining and Reclamation, Vancouver BC, Canada.

Silverman, M.P. \& Lundgren, D.C. (1959). Studies on the chemoautotrophic iron bacterium ferrobacillus ferrooxidans ii. Journal Bacteriol, 78(3), 326-331. https://bit.ly/2ImZhIW 